\title{
Gauge-boson scattering signals at the LHC
}

\author{
Suraj N. Gupta and James M. Johnson \\ Department of Physics, Wayne State University, Detroit, Michigan 48202 \\ Glenn A. Ladinsky and Wayne W. Repko \\ Department of Physics and Astronomy, Michigan State University, East Lansing, Michigan 48824
}

(October 3, 1995)

\begin{abstract}
We have extended our earlier treatment of the gauge-boson scattering with radiative corrections in the standard model at supercollider energies, and computed the rates for gauge-boson scattering modes in $p p$ collisions leading to the final states $W^{+} W^{-}, Z Z(4 l), Z Z(2 l 2 \nu), W^{ \pm} Z$, and $W^{ \pm} W^{ \pm}$. Our results at the LHC energy of $\sqrt{s}=14 \mathrm{TeV}$ for $m_{H}=1000 \mathrm{GeV}$ are compared with those recently obtained by Bagger et al. These results will be useful in the search for the Higgs bosons at supercollider energies as well as for experimentally distinguishing the standard model from non-minimal Higgs models.
\end{abstract}

13.85.Qk,14.80.Er,14.80.Gt

Typeset using REVTEX 


\section{INTRODUCTION}

Along with the search for the elusive Higgs bosons, efforts continue to be made to experimentally distinguish between the minimal (standard) and non-minimal Higgs models [1]. Bagger et al. [2,3] have provided an interesting approach to deal with this issue by analyzing the gauge-boson scattering signals in hadron supercollider experiments. They have shown that the relative frequencies of the gauge-boson scattering modes leading to the

final states $W^{+} W^{-}, Z Z(4 l), Z Z(2 l 2 \nu), W^{ \pm} Z$, and $W^{ \pm} W^{ \pm}$are significantly different in the standard model from those involving other electroweak symmetry breaking mechanisms.

Recently, we carried out precise calculations of the $W, Z$, and Higgs-boson scattering amplitudes in the standard model at supercollider energies with the inclusion of the full oneloop radiative corrections, and explored the detection of the Higgs boson through gaugeboson scattering in $p p$ collisions [4]. We also emphasized that the apparent violation of unitarity in scattering, encountered by some earlier authors, is not a consequence of the fact that the weak interactions become strong at supercollider energies. Rather, the Feynman expansion of the scattering operator yields non-unitary amplitudes at all energies, and the problem becomes accentuated at higher energies. The unitarity of our amplitudes was ensured by using the K-matrix as well as the Padé methods.

We shall now extend our earlier treatment, and use unitarized scattering amplitudes to compute the rates for the various gauge-boson scattering modes in $p p$ collisions at the LHC energy of $\sqrt{s}=14 \mathrm{TeV}$ for $m_{H}=1000 \mathrm{GeV}$. Our results for the standard model will be compared with those given by Bagger et al. [3]

\section{UNITARIZED GAUGE-BOSON SCATTERING AMPLITUDES}

In Ref. 4 , we calculated the $S$-wave amplitudes for the scattering of the $W, Z$, and Higgsbosons in the coupled neutral channels $W_{L}^{+} W_{L}^{-}, Z_{L} Z_{L}$ and $H H$. We have now extended our calculations by including the additional channels $W_{L}^{ \pm} Z_{L}$ and $W_{L}^{ \pm} W_{L}^{ \pm}$as well as the $P$ 
waves for all the channels. In view of an $\mathrm{SO}(3)$ symmetry associated with the gauge-boson interactions, all amplitudes are expressible in terms of three amplitudes $M, M^{\prime}$, and $M^{\prime \prime}$, which are given in Ref. 4.

The $l$-wave projection of the amplitude $M(s, t, u)$ is given by [5]

$$
a_{l}(s)=\frac{1}{32 \pi}\left(\frac{4\left|\mathbf{p}_{f}\right|\left|\mathbf{p}_{i}\right|}{s}\right)^{1 / 2} \int_{-1}^{1} d \cos \theta M(s, t, u) P_{l}(\cos \theta)
$$

and let $b(s), \bar{b}(s), c(s)$ and $d(s)$ denote similar projections of $M(t, s, u), M(u, t, s), M^{\prime}(s, t, u)$ and $M^{\prime \prime}(s, t, u)$, respectively. Then, the $l$-wave amplitude for the coupled $W_{L}^{+} W_{L}^{-}, Z_{L} Z_{L}$ and $H H$ channels can be expressed as

$$
a_{l}=\left(\begin{array}{ccc}
a_{l}+b_{l} & \frac{a_{l}}{\sqrt{2}} & \frac{c_{l}}{\sqrt{2}} \\
\frac{a_{l}}{\sqrt{2}} & \frac{1}{2}\left(a_{l}+b_{l}+\bar{b}_{l}\right) & \frac{c_{l}}{2} \\
\frac{c_{l}}{\sqrt{2}} & \frac{c_{l}}{2} & \frac{d_{l}}{2}
\end{array}\right),
$$

and

$$
\begin{aligned}
a_{l}\left(W_{L}^{ \pm} Z_{L} \rightarrow W_{L}^{ \pm} Z_{L}\right) & =b_{l}, \\
a_{l}\left(W_{L}^{ \pm} W_{L}^{ \pm} \rightarrow W_{L}^{ \pm} W_{L}^{ \pm}\right) & =\frac{1}{2}\left(b_{l}+\bar{b}_{l}\right),
\end{aligned}
$$

where we have introduced appropriate factors to eliminate the identical-particle restrictions on the $W_{L}^{ \pm} W_{L}^{ \pm}, Z_{L} Z_{L}$, and $H H$ phase spaces.

The above amplitudes, obtained from Feynman's expansion of the scattering operator, require unitarization, and, as explained in Ref. 4, we have used the K-matrix and Padé methods for this purpose. The K-matrix amplitude for the lth partial wave is given by

$$
a_{l}^{K}=\frac{a_{l}^{(1)}+\operatorname{Re} a_{l}^{(2)}}{1-i\left(a_{l}^{(1)}+\operatorname{Re} a_{l}^{(2)}\right)},
$$

while the Padé amplitude is

$$
a_{l}^{P}=\frac{a_{l}^{(1)^{2}}}{a_{l}^{(1)}-a_{l}^{(2)}}
$$


where $a_{l}^{(1)}$ and $a_{l}^{(2)}$ denote the tree and one-loop amplitudes. When $a_{l}$ is a matrix for multichannel scattering, (5) and (6) take the forms

$$
\begin{aligned}
& a_{l}^{K}=\left(a_{l}^{(1)}+\operatorname{Re} a_{l}^{(2)}\right)\left[1-i\left(a_{l}^{(1)}+\operatorname{Re} a_{l}^{(2)}\right)\right]^{-1}, \\
& a_{l}^{P}=a_{l}^{(1)}\left(a_{l}^{(1)}-a_{l}^{(2)}\right)^{-1} a_{l}^{(1)} .
\end{aligned}
$$

We have plotted in Fig. 1 absolute values of the K-matrix and the Padé amplitudes for scatterings among the various gauge-boson channels as functions of the center-of-mass energy $\sqrt{s}$ for $m_{H}=1000 \mathrm{GeV}$. Both the $S$ - and $P$-wave amplitudes are shown, and it may be noted that the $P$-wave amplitudes vanish for states containing identical gauge bosons. The K-matrix and the Padé amplitudes agree at lower energies, but the Padé amplitudes seem to exhibit an interesting but odd behavior at higher energies. In particular, striking $P$ wave resonances in the $W_{L}^{+} W_{L}^{-}$and $W_{L}^{ \pm} Z_{L}$ channels appear in the Padé amplitudes around $\sqrt{s}=3000 \mathrm{GeV}[6]$. The physical significance of the Padé amplitude resonances has been analyzed in general by Atkinson, Harada, and Sanda [7].

\section{GAUGE-BOSON PAIR PRODUCTION VIA FUSION IN $p p$ COLLISIONS}

We have used the unitarized amplitudes to compute the invariant-mass distributions for gauge-boson pair production via fusion in $p p$ collisions at the LHC energy of $\sqrt{s}=14 \mathrm{TeV}$ for $m_{H}=1000 \mathrm{GeV}$. Our calculations are based on the effective-W approximation [8], and we have used the same cuts and backgrounds as proposed by Bagger et al. The distributions for production modes leading to the final states $W^{+} W^{-}, Z Z(4 l), Z Z(2 l 2 \nu), W^{ \pm} Z$, and $W^{ \pm} W^{ \pm}$are shown in Fig. 2.

We have also computed the event rates per LHC-year for gauge-boson fusion signals, assuming $\sqrt{s}=14 \mathrm{TeV}$ and an annual luminosity of $100 \mathrm{fb}^{-1}$. Our results for the standard model, obtained with the K-matrix and the Padé unitarizations, are compared with those of Bagger et al. in Table I. It is interesting that the K-matrix and Padé unitarization results are practically the same for all final states despite the appearance of the $P$-wave resonances 
in the channels leading to the $W_{L}^{+} W_{L}^{-}$and $W_{L}^{ \pm} Z_{L}$ states.

In Ref. 3, it was shown that models for strongly interacting symmetry breaking with a scalar isospin-zero resonance (standard model, scalar model and $\mathrm{O}(2 \mathrm{~N})$ model) will yield a large excess of events in the $Z_{L} Z_{L} \rightarrow 2 l 2 \nu, Z_{L} Z_{L} \rightarrow 4 l$, and $W_{L}^{+} W_{L}^{-} \rightarrow 2 l 2 \nu$ final states, while models with a heavy isospin-one vector resonance, or no resonance at all, will provide a large enhancement of the $W_{L}^{ \pm} W_{L}^{ \pm} \rightarrow 2 l 2 \nu$ final states. It is the distinguishing features of these models, examined from the perspective of all the various final states, that allows different mechanisms for strongly interacting electroweak symmetry breaking to be recognized.

Our standard-model calculations have produced some differences with respect to the previous analysis of the electroweak symmetry breaking sector given in Ref. 3. The results computed here demonstrate an enhancement of the $W_{L}^{ \pm} W_{L}^{ \pm}$final states, bringing the event rate closer to that of the vector models with a heavier resonance. Although this increase to about 8 events/LHC-year is still smaller than the isovector and nonresonant enhancements computed in Ref. 3, it can still increase the expected time it may take to distinguish these symmetry breaking mechanisms from the standard model by a couple of years.

Through the application of both the K-matrix and Padé unitarizations, we find some decrease in the event rates for the $W_{L}^{+} W_{L}^{-}, Z_{L} Z_{L}(4 l)$, and $Z_{L} Z_{L}(2 l 2 \nu)$ modes. This does not significantly affect the usefulness of these modes in distinguishing the standard model or $\mathrm{O}(2 \mathrm{~N})$ models from the other mechanisms of spontaneous symmetry breaking; there is only an indication that the separation between the chiral lagrangian model with a heavy scalar resonance and the standard model becomes less clear.

As for the production of $W_{L}^{ \pm} Z_{L}$ pairs, with the K-matrix or the Padé unitarization there is no significant change from the results of Ref. 3.

Since the standard model is the most popular and economical of all the models, it is desirable to obtain the standard model results as precisely as possible. Our theoretical results will be useful in the search for the Higgs bosons at supercollider energies as well as 
for experimentally distinguishing the standard model from non-minimal Higgs models.

\section{ACKNOWLEDGMENTS}

This work was supported in part by the U.S. Department of Energy under Grant

No. DE-FG02-85ER40209 and the National Science Foundation under Grant Nos. PHY93-07980 and PHY-93-19216. 


\section{REFERENCES}

[1] J. F. Gunion, H. E. Haber, G. Kane, and S. Dawson, The Higgs Hunter's Guide, (Addison-Wesley, New York, 1990).

[2] J. Bagger, V. Barger, K. Cheung, J. Gunion, T. Han, G. A. Ladinsky, R. Rosenfeld, and C.-P. Yuan, Phys. Rev. D 49, 1246 (1994).

[3] J. Bagger, V. Barger, K. Cheung, J. Gunion, T. Han, G. A. Ladinsky, R. Rosenfeld, and C.-P. Yuan, hep-ph/9504426. (To appear in Physical Review D)

[4] S. N. Gupta, J. M. Johnson and W. W. Repko, Phys. Rev. D 48, 2083 (1993). Extensive references to earlier work are cited in this paper. Note that in the formula for the integral $B_{3}(s)$, given in the Appendix of the paper, " $2-\frac{2 \pi}{\sqrt{3}}$ for $s=1$ " should read " $2-\frac{\pi}{\sqrt{3}}$ for $s=1 "$.

[5] L. Durand, J. M. Johnson, and J. L. Lopez, Phys. Rev. Lett. 64, 1215 (1990); Phys. Rev. D 45, 3112 (1992). The kinematic factor $\left(4\left|\mathbf{p}_{f}\right|\left|\mathbf{p}_{i}\right| / s\right)^{1 / 2}$ in Eq. (1) reduces to unity for the gauge-boson channels and to $(1-4 / s)^{1 / 2}$ for the $H H$ channel.

[6] In the $W_{L}^{+} W_{L}^{-}$channel in the Padé amplitude, a $P$-wave resonance was also shown to appear in an earlier investigation by D. A. Dicus and W. W. Repko, Phys. Rev. D 47, 4154 (1993).

[7] D. Atkinson, M. Harada, and A. I. Sanda, Phys. Rev. D 46, 3884 (1992).

[8] M. S. Chanowitz and M. K. Gaillard, Phys. Lett. B 142, 85 (1984); G. L. Kane, W. W. Repko, and W. B. Rolnick, Phys. Lett. B 148, 367 (1984); S. Dawson, Nucl. Phys. B249, $42(1985)$. 


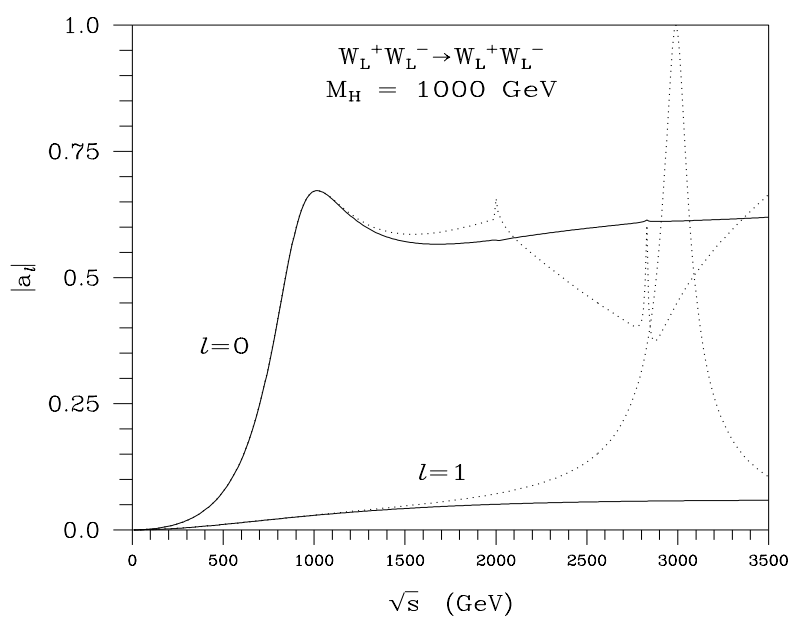

(a)

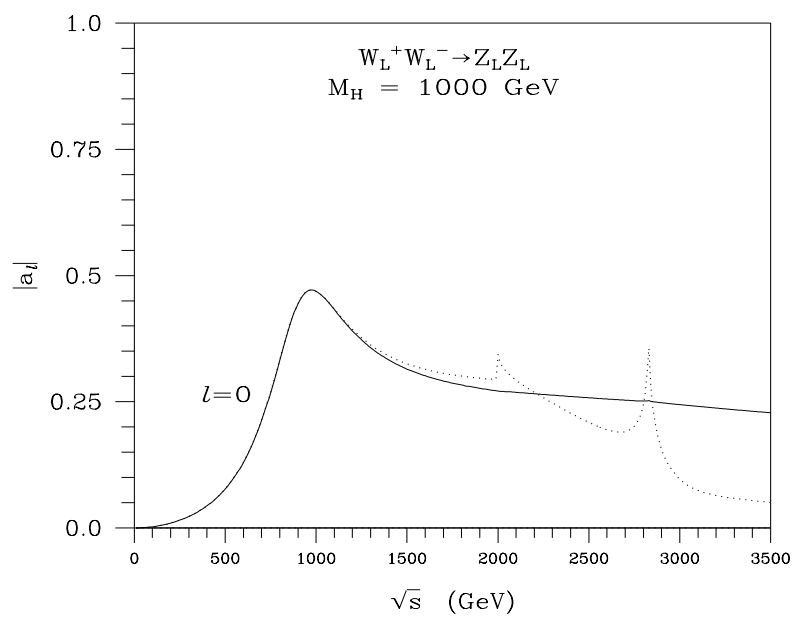

(c)

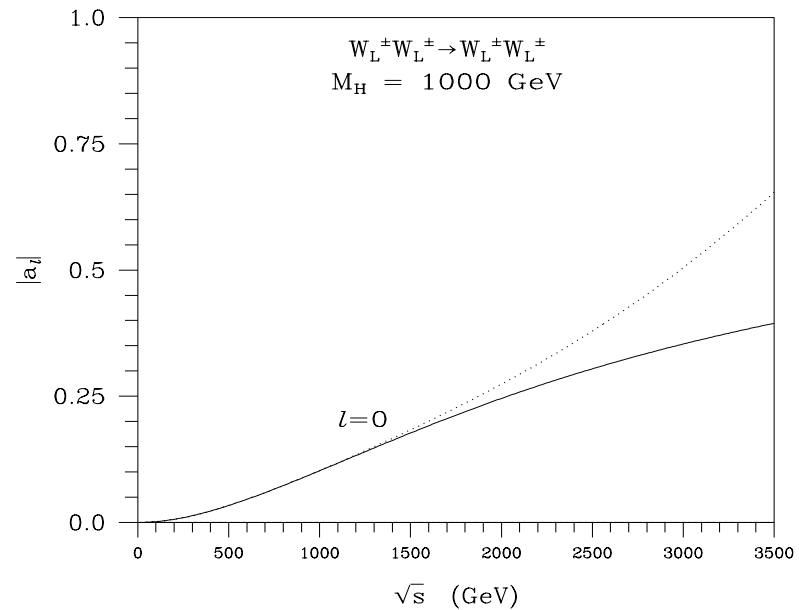

(e)

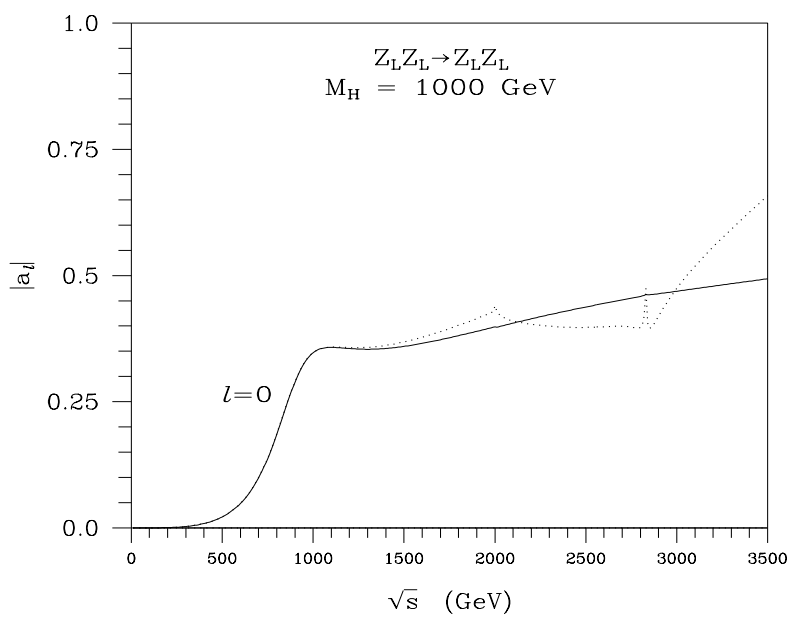

(b)

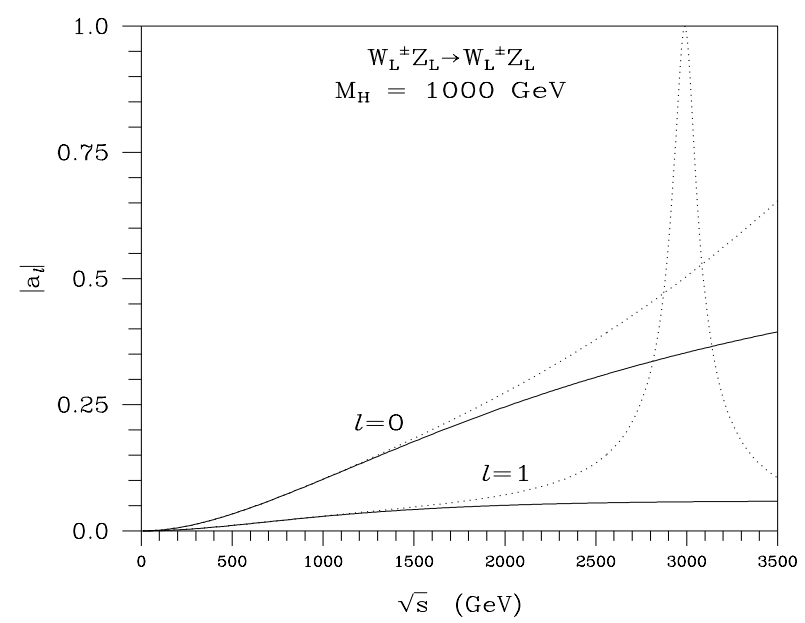

(d)

FIG. 1. Absolute values of the $S$ - and $P$-wave amplitudes for scattering among the gauge-boson channels as functions of the center-of-mass energy $\sqrt{s}$ for $m_{H}=1000 \mathrm{GeV}$. The solid lines represent the K-matrix amplitudes, and the dotted lines the Padé amplitudes. Note that $P$-wave amplitudes vanish for states containing identical gauge-bosons. 


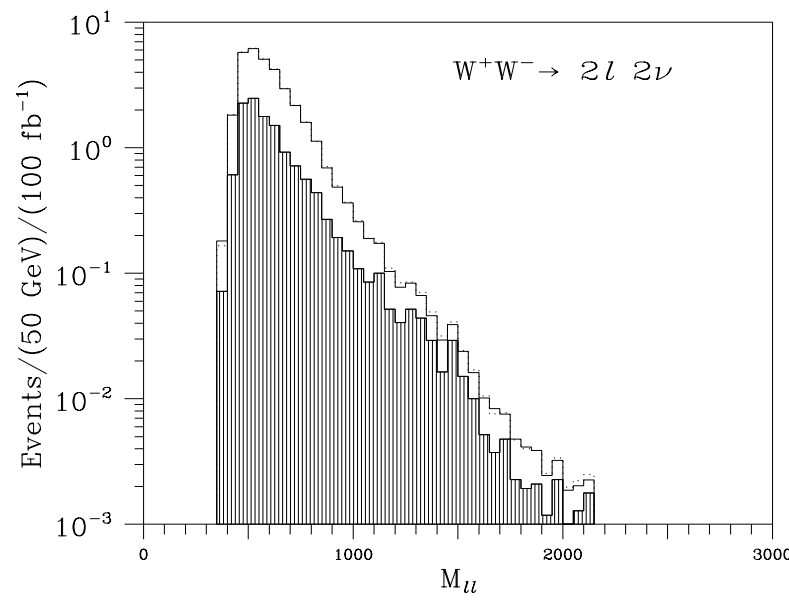

(a)

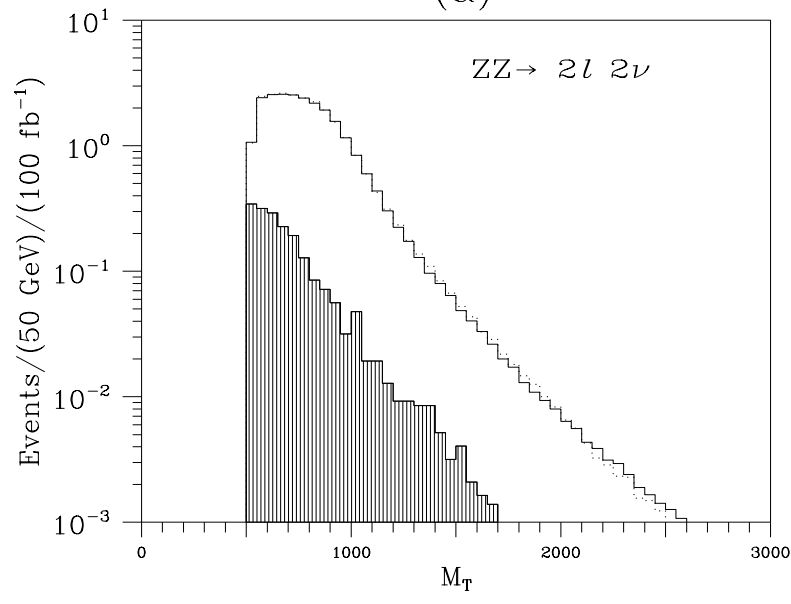

(c)

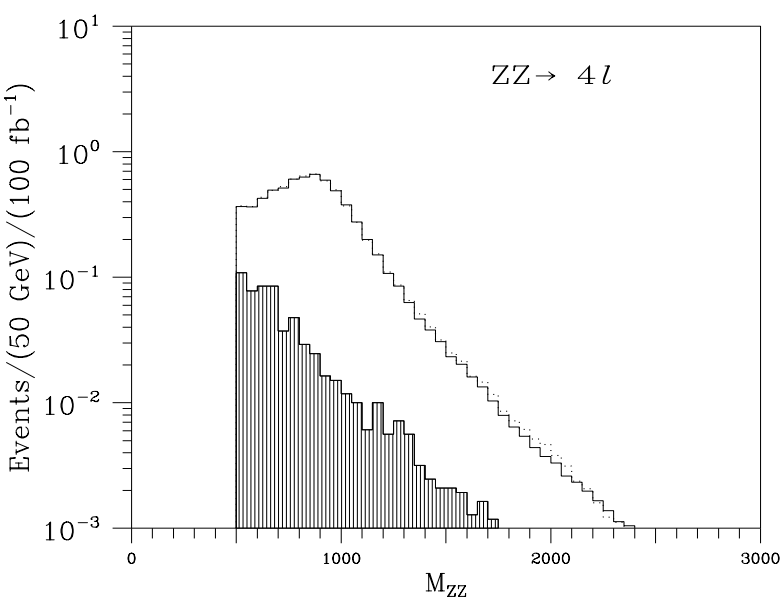

(b)

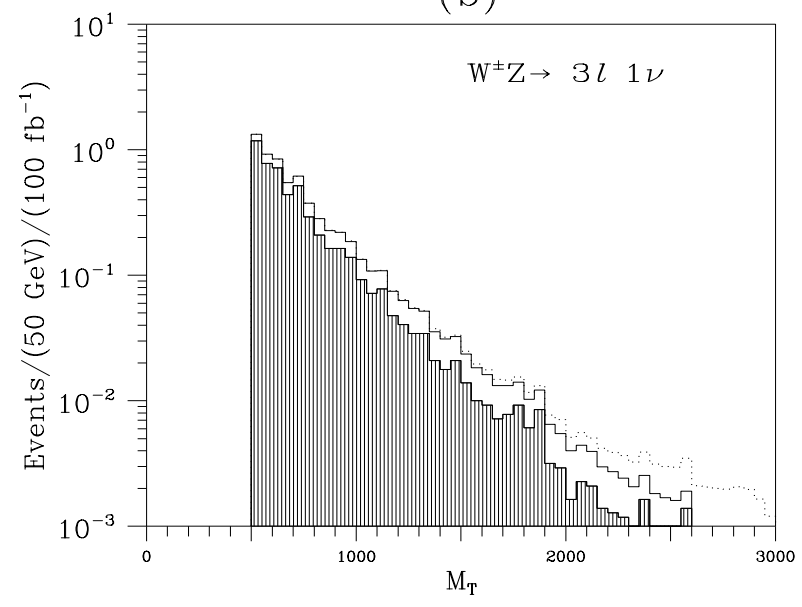

(d)

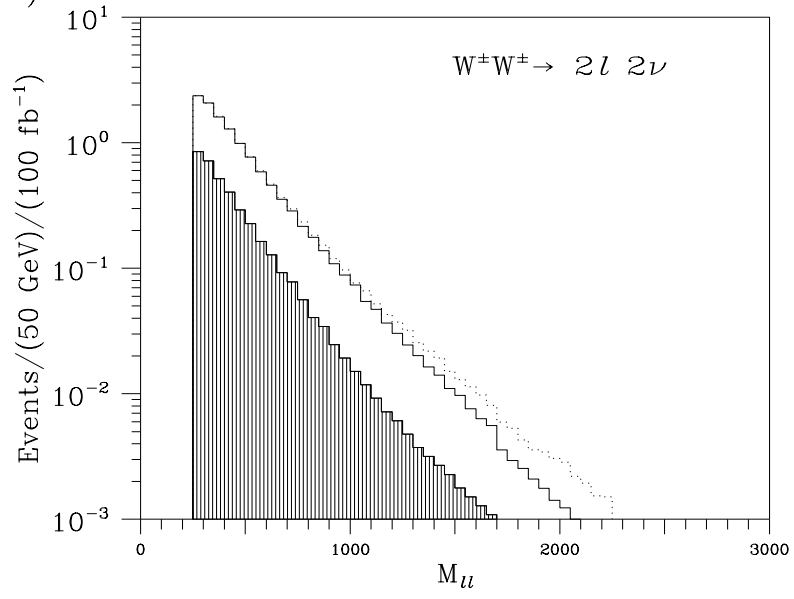

(e)

FIG. 2. Invariant mass distributions for the purely leptonic final states arising from the processes $p p \rightarrow W^{+} W^{-} X \rightarrow 2 l 2 \nu X, p p \rightarrow Z Z X \rightarrow 4 l X, p p \rightarrow Z Z X \rightarrow 2 l 2 \nu X$, $p p \rightarrow W^{ \pm} Z X \rightarrow 3 l \nu X$ and $p p \rightarrow W^{ \pm} W^{ \pm} X \rightarrow 2 l 2 \nu X$ for $\sqrt{s}=14 \mathrm{GeV}$ and an annual LHC luminosity of $100 \mathrm{fb}^{-1}$. The K-matrix and Padé signals for the standard model with $m_{H}=1000 \mathrm{GeV}$ are shown as solid and dotted lines above the background, and plotted as the number of events per $50 \mathrm{GeV}$ bin, per $100 \mathrm{fb}^{-1} . M_{l l}$ is the invariant mass of the experimentally observed leptons, $M_{Z Z}$ is the invariant mass of the $Z Z$ pair, and $M_{T}$ is the cluster transverse mass as defined in Ref. 3 . 
TABLE I. Event rates per LHC-year for gauge-boson fusion, together with backgrounds, assuming $\sqrt{s}=14 \mathrm{TeV}$ and an annual luminosity of $100 \mathrm{fb}^{-1}$. Our cuts and backgrounds are the same as those of Bagger et al.

\begin{tabular}{lcccc}
\hline \hline Final state & Bkgd. & Bagger et. al & $\begin{array}{c}\text { K-Matrix } \\
\text { unitarization }\end{array}$ & $\begin{array}{c}\text { Padé } \\
\text { unitarization }\end{array}$ \\
\hline$W^{+} W^{-}$ & 12 & 27 & 21 & 21 \\
$Z Z(4 l)$ & 0.7 & 9 & 6.1 & 6.1 \\
$Z Z(2 l 2 \nu)$ & 1.8 & 29 & 21 & 22 \\
$W^{ \pm} Z$ & 4.9 & 1.2 & 1.3 & 1.3 \\
$W^{ \pm} W^{ \pm}$ & 3.7 & 5.6 & 8.2 & 8.4 \\
\hline \hline
\end{tabular}

